\title{
Editorials
}

\section{Perioperative blood conservation - The experts, the elephants, the clinicians, and the gauntlet}

\author{
Keyvan Karkouti MD, ${ }^{*} \dagger$ Stuart A. McCluskey MD*
}

$\mathrm{P}$ RACTITIONERS of perioperative blood conservation aim to reduce, or eliminate, the need for allogeneic blood transfusion during surgery. Widespread interest in this field followed the blood-borne infection epidemic of the 1970s and 1980s, during which thousands of Canadians received tainted blood and were infected with the human immunodeficiency and hepatitis viruses. Interest in perioperative blood conservation remains strong even though the viral-infectious risks of allogeneic blood, as well as its other explicit serious risks that include major hemolytic reactions, transfusion associated acute lung injury, and bacterial contamination, are exceedingly small. ${ }^{1,2}$ Proponents of perioperative blood conservation argue that minimizing allogeneic blood transfusion remains an important objective because allogeneic blood is expensive, is often in short supply, and may, owing to its immunomodulatory effects, increase morbidity and mortality. ${ }^{3}$

Some of the modalities employed for perioperative blood conservation include:

- Preoperative autologous blood donation, whereby the patient's blood is collected about two to four weeks before surgery for possible use by the patient during surgery.

- Preoperative treatment with erythropoiesis-stimulating agents (ESAs), to raise patients' red blood cell mass to allow a greater tolerance for blood loss before allogeneic blood is needed.

- Intraoperative treatment with antifibrinolytic drugs, which reduce blood loss and allogeneic blood transfusion by alleviating excessive fibrinolysis during surgery.
- Intraoperative acute normovolemic hemodilution, whereby patients' blood is collected early in the course of surgery, is replaced with crystalloids or colloids to maintain euvolemia, and is re-infused to the patient near the end of surgery. By rendering patients anemic during the blood loss phase of surgery, this intervention reduces the number of red blood cells that are lost, which may reduce the need for allogeneic blood transfusions.

The past year has been particularly interesting for practitioners of perioperative blood conservation, for while a much-anticipated clinical practice guideline expressing the opinions of many of the experts in the field was released, new safety concerns with prominent blood conservation modalities were identified that raise fundamental questions about the basic tenets of the field. Herewith we will outline these and other recent developments in this field, explore how they relate to clinical practice, and outline why, in our opinion, they expose critical shortcomings in our current state of knowledge of the risk-benefit profile of available blood conservation modalities.

\section{The experts}

In May 2007, the Society of Thoracic Surgeons and the Society of Cardiovascular Anesthesiologists published an authoritative review and clinical practice guidelines for perioperative blood conservation in cardiac surgery. ${ }^{4}$ While their review and recommendations were exhaustive in breadth, their principal recommendation was that clinicians should employ all available measures of blood conservation in patients who are predicted to be at high-risk for receiving peri-

CAN J ANESTH 2007 / 54: $11 /$ pp 861-867

From the Departments of Anesthesia* and Health Policy, Management, and Evaluation, $†$ University Health Network, University of Toronto, Toronto, Ontario, Canada.

Address correspondence to: Dr. Keyvan Karkouti, University Health Network, Toronto General Hospital, Department of Anesthesia, EN 3-402, 200 Elizabeth Street, Toronto, Ontario M5G 2C4, Canada. Phone: 416-340-5164; Fax: 416-340-3698;

E-mail: keyvan.karkouti@uhn.on.ca

Competing interests: The authors have received research funding from the following pharmaceutical companies involved in perioperative transfusion management: Novo Nordisk, Bayer, Ortho Biotech, Bristol-Myers Squibb, and Fresenius Kabi. 
operative blood transfusion. To that end, they recommended the use of antifibrinolytic drugs, predonation of autologous blood, preoperative administration of ESAs, intraoperative cell salvage, and acute normovolemic hemodilution.

In a more general review of the topic, Doctors Goodnough and Shander, two eminent experts in the field, also recommended that clinicians use multiple blood management modalities to minimize or eliminate allogeneic transfusions in all patients undergoing surgery. ${ }^{5}$

There is, therefore, a consensus among the experts that the benefits of multi-modality blood conservation strategies aimed at reducing or eliminating allogeneic blood transfusions during surgery outweigh the combined risks (and presumably the costs) of the recommended blood conservation modalities. Three recent developments, however, have raised serious questions about the validity of this assumption.

\section{The elephants}

One recent development is the recognition of new safety concerns with two of the most prominent and extensively studied blood conservation modalities, aprotinin and ESAs.

Recently, several observational studies and a metaanalysis of randomized controlled trials have shown that aprotinin is associated with increased risk of renal dysfunction. ${ }^{6-8}$ Whether aprotinin causes renal toxicity or a benign, transient biochemical abnormality, however, is untested and a matter of much controversy. Nonetheless, these new findings led the US Food and Drug Administration (FDA) to restrict the indications for aprotinin to high-risk coronary artery bypass surgery and to highlight its safety warnings to include its proclivity for causing renal dysfunction and possibly renal failure. ${ }^{\mathrm{A}}$ It is interesting to note that these events occurred fully 13 years after the FDA initially approved aprotinin for perioperative blood conservation.

Similarly, recently identified safety concerns led the FDA to highlight its safety warnings for ESAs, coincidentally also 13 years after it initially approved them for perioperative use to reduce transfusion requirements. ${ }^{\mathrm{B}}$ One concern is that perioperative use of ESAs may increase the risk of thromboembolic events. In a recently completed large $(n=681)$ randomized controlled trial, anemic patients receiving ESAs prior to spinal surgery had a significantly higher rate of deep vein thrombosis compared with those who received standard transfusion support $(4.7 \%$ vs $2.1 \%) .{ }^{\mathrm{C}}$ One major limitation of this study was that patients did not receive prophylactic anticoagulation against deep vein thrombosis, which may protect against the thrombo- embolic risks of ESAs. ${ }^{9}$ Also of concern is that ESAs may also increase the risk of other serious adverse events and death when they are used to treat or prevent anemia in cancer patients. ${ }^{10}$ Whether short-term, perioperative use of these drugs for perioperative blood conservation in cancer surgery is similarly harmful, however, has not been studied.

While these new safety issues with aprotinin and erythropoietin are concerning in and of themselves, they also highlight the fact that definitive safety data are lacking for all blood conservation modalities, even those with exhaustive and indisputable efficacy data.

A second recent development is the recognition that none of the blood conservation modalities has been shown to reduce morbidity and mortality. Metaanalytical analyses of randomized placebo-controlled clinical trials of antifibrinolytic drugs, for example, have shown that despite being highly efficacious in reducing blood loss, blood transfusion, and re-exploration in cardiac surgery, they do not improve patient outcomes. ${ }^{8}$ If allogeneic blood transfusions are indeed harmful, then why do these highly efficacious drugs not improve outcomes? One possible explanation is that their efficacy is too modest - on average, they reduce blood loss by less than $500 \mathrm{~mL}$ in cardiac surgery ${ }^{8}$ to translate into improved outcomes. If true, this would suggest that low-volume blood loss necessitating the transfusion of one or two units of blood is not harmful. This would be consistent with the findings of recent laboratory and observational studies that what is harmful is large-volume blood loss that leads to the transfusion of multiple units of blood products. ${ }^{11-13}$ However, there is no evidence that standard multi-modality blood conservation strategies can prevent large-volume blood loss.

Another possible explanation why none of the blood conservation modalities has been shown to improve morbidity and mortality is that their effects on outcomes other than allogeneic blood transfusion have not been properly tested. For the most part, these modalities have been tested by small trials that excluded high-risk cases, rendering them underpowered (both individually and collectively) for detecting the effects (positive or negative) of the modalities on morbidity and mortality. A third possible explanation is that the benefits of blood conservation modalities

\footnotetext{
A http://www.fda.gov/bbs/topics/NEWS/2006/NEW01529. html; accessed 3 July 2007.

B http://www.fda.gov/cder/drug/infopage/RHE/default.htm; accessed 3 July 2007.

C http://download.veritasmedicine.com/PDF/CR004621_CSR. pdf; accessed 3 July 2007.
} 
on clinical outcomes are counteracted by their unmeasured or undetected harms.

A third recent development is the publication of a randomized, multicentre controlled clinical trial that raises questions about how effective (rather than efficacious) multi-modality blood conservation strategies are in routine clinical practice. In recommending the use of multi-modality blood conservation strategies, clinical guidelines often cite studies that found such strategies to be highly efficacious in reducing exposure to allogeneic blood transfusions. ${ }^{14,15}$ These and other similar studies, however, have important limitations - often, they are small, single-centre studies that lack proper controls or blinding - that may limit their generalizability. In the first rigorous examination of such strategies, Feagan and associates randomized 30 hospitals to implement a multi-modality blood conservation strategy or to continue with standard care (which included the use of autologous predonation in about $25 \%$ of cases) in orthopedic surgery patients. ${ }^{16}$ They found that the intervention was only modestly successful, in that it reduced exposure to allogeneic blood transfusion by about $10 \%$ (from $26.1 \%$ to $16.5 \%)$. Thus, this study revealed that multi-modality blood conservation strategies are effective, but not to the extent that is assumed by clinical guidelines that recommend their use.

\section{The clinicians}

The basic tenet of clinical medicine is, first, do no harm. ${ }^{17}$ Consequently, the decision to employ any perioperative blood conservation modality, as in other medical interventions, should be based on a careful risk-benefit analysis. ${ }^{17}$ Practitioners of perioperative medicine, therefore, have a dilemma. On the one hand, they are advised by experts to employ multimodality blood conservation strategies to reduce exposure to perioperative allogeneic blood transfusions. On the other hand, the safety and effectiveness of blood conservation modalities have not been adequately tested; thus, it is not known if their risks, whether used singly or in various combinations, are outweighed by their benefits.

Given that there is no reliable data on the riskbenefit profiles of blood conservation modalities, how can one explain the wide consensus among the experts for their continued use? In our opinion, a corollary can be made with how experts responded to the threat of Creutzfeldt-Jakob disease (vCJD) to the blood system. Studies have found that the approach to this threat was heavily influenced by the catastrophic events of the blood-borne infection epidemic of the 1970s and 1980s, resulting in the adoption of safety measures with relative high cost-effectiveness in advance of definitive evidence (i.e., the precautionary approach). ${ }^{18,19}$ Whereas this approach may arguably have been appropriate to protect the safety of the blood system, we believe that it is inappropriate for perioperative blood conservation, where each modality has inherent risks that may be several fold greater than the current proven risks of allogeneic blood transfusion.

\section{The gauntlet}

To solve the clinicians' dilemma, we challenge researchers and research-based pharmaceutical companies involved in this field to design and carry out adequately powered randomized controlled clinical trials to determine the risk-benefit profile of multi-modality strategies for perioperative blood conservation. This, we recognize, will be difficult to do, as it will require large or complex, expensive trials. Nonetheless, such trials have been carried out in other fields, ${ }^{20}$ and are being carried out in this field as well. ${ }^{21}$ Until such trials are carried out, it would be prudent for clinicians employing blood conservation modalities to fully inform their patients of the potential risks of the modalities being employed and to clearly stipulate to the patient that these risks may outweigh any potential benefits of avoiding allogeneic blood transfusions. The opinion of experts notwithstanding, it would be still more prudent to limit the use of most blood conservation modalities to the research setting until their risk-benefit profile is clearly determined.

\section{La conservation péri- opératoire du sang : les experts, les éléphants, les cliniciens et le gant}

Les praticiens chargés de la conservation périopératoire du sang ont pour but de réduire, voire d'éliminer, les besoins transfusionnels de sang allogène pendant la chirurgie. Un intérêt global pour ce domaine est survenu à la suite des épidémies infectieuses transmises par le sang survenues dans les années 70 et 80 , période durant laquelle des milliers de Canadiens ont reçu du sang contaminé et ont été infectés par les virus de l'immunodéficience humaine et de l'hépatite. 
L'intérêt pour la conservation périopératoire du sang demeure d'actualité, bien que les risques d'infection virale du sang allogène, ainsi que les autres risques explicites sévères - y compris les réactions hémolytiques importantes, les lésions pulmonaires aiguës liées à la transfusion et la contamination bactérienne - soient minimes. ${ }^{1,2}$ Les partisans de la conservation périopératoire du sang avancent que la réduction des transfusions de sang allogène au plus bas niveau demeure un objectif primordial. En effet, le sang allogène est cher, vient souvent à manquer et peut augmenter la morbidité et la mortalité en raison de ses propriétés immunomodulatrices. ${ }^{3}$

Parmi les modalités utilisées pour la conservation périopératoire du sang, citons :

- Le don autologue préalable : le sang du patient est récolté deux à quatre semaines avant l'opération, au cas où le patient en aurait besoin pendant la chirurgie.

Le traitement préopératoire à base d'agents stimulateurs d'érythropoïèse (ASE) : ce procédé permet d'augmenter la masse de globules rouges des patients, ce qui favorise une meilleure tolérance aux pertes sanguines avant que du sang allogène soit nécessaire.

- Le traitement peropératoire à base d'agents antifibrinolytiques, lesquels réduisent la perte sanguine et les transfusions de sang allogène en modérant une fibrinolyse excessive pendant la chirurgie.

L'hémodilution normovolémique aiguë peropératoire : le sang du patient est récolté au début de la chirurgie, est remplacé par des agents cristalloïdes et colloïdes pour maintenir l'euvolémie, et est reperfusé au patient vers la fin de la chirurgie. Parce que cette intervention rend les patients anémiques pendant la phase de perte sanguine de la chirurgie, elle réduit la perte de globules rouges, ce qui peut, à son tour, réduire le besoin en transfusions de sang allogène.

Cette dernière année s'est révélée particulièrement intéressante pour les praticiens de la conservation périopératoire du sang. En effet, tandis que les recommandations très attendues pour la pratique clinique exprimant les opinions de nombreux experts du domaine étaient publiées, de nouvelles préoccupations sur la sécurité d'importantes modalités de conservation du sang ont été identifiées. Ces préoccupations soulèvent des questions fondamentales à propos des principes de base de ce domaine. Nous allons ici esquisser ces développements récents ainsi que d'autres dans ce domaine, puis nous explorerons leurs liens avec la pratique clinique ; enfin, nous exposerons brièvement pourquoi, selon nous, ces développements mettent en lumière les insuffisances cruciales de nos connaissances actuelles du profil risque/bénéfice des modalités de conservation du sang disponibles à ce jour.

\section{Les experts}

En mai 2007, la Société des chirurgiens thoraciques et la Société des anesthésiologistes cardiovasculaires ont publié un article de synthèse accompagné de recommandations pour la pratique clinique faisant autorité au sujet de la conservation périopératoire du sang en chirurgie cardiaque. ${ }^{4}$ Tout en étant exhaustifs dans leur synthèse et leurs recommandations, ces experts recommandent tout d'abord aux cliniciens d'avoir recours à toutes les mesures disponibles de conservation du sang pour les patients chez qui on prévoit un risque élevé de recevoir des transfusions sanguines avant, pendant ou après l'intervention. Pour ce faire, ils recommandent l'utilisation d'agents antifibrinolytiques, le don préalable de sang autologue, l'administration préopératoire d'ASE, la préservation des globules durant l'opération et une hémodilution normovolémique aiguë.

Dans une synthèse plus générale du sujet, les docteurs Goodnough et Shander, deux experts reconnus du domaine, recommandent également aux cliniciens le recours à plusieurs modalités de prise en charge du sang afin de minimiser, voire éliminer, les transfusions allogènes pour tous les patients subissant une chirurgie. ${ }^{5}$

Ainsi, il existe un consensus parmi les experts quant aux bénéfices de stratégies multimodales de conservation du sang qui visent à réduire ou à éliminer les transfusions de sang allogène pendant la chirurgie ; en effet, ces bénéfices sont plus importants que les risques combinés (et sans doute les coûts) des modalités de conservation du sang recommandées. Toutefois, trois développements récents ont soulevé des questions sérieuses quant à la validité de cette hypothèse.

\section{Les éléphants}

L'un des développements récents est la reconnaissance de nouvelles préoccupations quant à la sécurité de deux des modalités de conservation du sang les plus importantes et les plus approfondies : l'aprotinine et les ASE.

Récemment, de nombreuses études d'observation ainsi qu'une méta-analyse d'études randomisées contrôlées ont montré que l'aprotinine est associée à un risque accru d'insuffisance rénale. ${ }^{6-8}$ Toutefois, on n'a pas testé si l'aprotinine provoque une toxicité rénale ou une anomalie biochimique bénigne et de courte durée, et ces données sont controversées. Néanmoins, ces nouveaux éléments ont poussé la Food and Drug Administration (FDA) américaine à restreindre les 
indications de l'aprotinine à la chirurgie de pontage aortocoronarien à haut risque ainsi qu'à souligner ses mises en garde de sécurité pour qu'ils incluent la propension de l'aprotinine à causer une dysfonction rénale, voire une insuffisance rénale. ${ }^{A}$ Il est intéressant de noter que tout ceci est arrivé 13 ans après que la FDA a pour la première fois approuvé l'aprotinine pour la conservation périopératoire du sang.

De même, des préoccupations sanitaires récemment identifiées ont mené la FDA à souligner ses mises en garde de sécurité pour les ASE et ce, également 13 ans après leur approbation initiale pour une utilisation périopératoire afin de réduire les besoins transfusionnels. ${ }^{\mathrm{B}}$ Une des préoccupations provient du fait que l'utilisation périopératoire d'ASE pourrait augmenter le risque d'événements thromboemboliques. Dans une récente étude randomisée contrôlée à large échelle $(n$ $=681$ ), les patients anémiques recevant des ASE avant une chirurgie rachidienne ont présenté un taux significativement plus élevé de thrombose veineuse profonde que les patients ayant reçu un traitement transfusionnel standard. (4,7 \% vs $2,1 \%) .{ }^{\mathrm{C}}$ Une des limites majeures de cette étude est que les patients n'ont pas reçu d'agents anticoagulants prophylactiques contre la thrombose veineuse profonde, ce qui pourrait protéger les patients des risques thromboemboliques liés aux ASE. ${ }^{9}$ Il est également préoccupant que les ASE puissent augmenter le risque d'autres effets secondaires sérieux et de décès lorsqu'ils sont utilisés pour traiter ou prévenir l'anémie chez les patients cancéreux. ${ }^{10}$ Cependant, il n'existe pas d'étude déterminant si l'utilisation périopératoire à court terme de ces médicaments pour la conservation périopératoire du sang dans les chirurgies du cancer est tout aussi néfaste.

Bien que ces nouvelles questions d'innocuité de l'aprotinine et de l'érythropoiétine soient préoccupantes en soi et pour elles-mêmes, elles soulignent également le fait que des données de sécurité définitives manquent encore pour toutes les modalités de conservation du sang, même pour celles possédant des données complètes et indiscutables quant à leur efficacité.

Un deuxième développement récent a trait à la prise de conscience qu'aucune des modalités de conservation du sang n'a d'effet démontré sur la réduction de la morbidité et de la mortalité. Des analyses méta-analytiques d'études cliniques randomisées et contrôlées par placebo d'agents antifibrinolytiques, par exemple, ont montré que, malgré leur efficacité élevée dans la réduction des pertes sanguines, de la transfusion de sang et de la ré-exploration en chirurgie cardiaque, ces agents n'améliorent pas le devenir des patients. ${ }^{8}$ Si les transfusions de sang allogène sont véritablement nocives, alors pourquoi ces médicaments hautement efficaces n'améliorent pas les devenirs ? Une explication possible est que leur efficacité est trop modeste : en moyenne, ils réduisent la perte sanguine de moins de $500 \mathrm{~mL}$ en chirurgie cardiaque ${ }^{8}$, trop peu pour se traduire en devenirs améliorés. Si c'est le cas, cela suggère qu'une perte sanguine de bas volume ne nécessitant la transfusion que d'une ou deux unités de sang n'est pas nocive, ce qui serait en accord avec les résultats de récentes études de laboratoire et d'observation qui déduisent que ce sont les larges pertes sanguines menant à la transfusion de nombreux produits sanguins qui sont nocives. ${ }^{1-13}$ Toutefois, aucune donnée ne suggère que les stratégies de conservation du sang multimodales standard puissent prévenir les pertes sanguines importantes.

Une autre explication plausible de la raison pour laquelle aucune des modalités de conservation du sang n'a été prouvée efficace dans l'amélioration de la morbidité et de la mortalité est que leurs effets sur les devenirs autres que la transfusion de sang allogène n'ont pas été testés de manière adéquate. La plupart de ces modalités ont été testées dans de petites études qui excluaient les cas à risque élevé, manquant ainsi de puissance (aussi bien sur le plan individuel que collectif) pour la détection des effets (positifs ou négatifs) des modalités sur la morbidité et la mortalité. Une troisième explication possible est que les bénéfices des modalités de conservation du sang sur les devenirs cliniques sont contrebalancés par leurs effets néfastes, lesquels ne sont ni mesurés ni détectés.

La publication d'une étude clinique randomisée, multi-centrique et contrôlée qui soulève des questions quant à l'efficacité (plutôt qu'à l'efficience) des stratégies multimodales de conservation du sang dans la pratique clinique de routine constitue un troisième développement récent. Dans leurs recommandations au sujet de l'utilisation de stratégies multimodales de conservation du sang, les directives cliniques citent souvent des études qui ont démontré que de telles stratégies étaient très efficaces dans la réduction de l'exposition des patients aux transfusions de sang allogène. ${ }^{14,15}$ Ces études - ainsi que d'autres allant dans la même direction - comportent toutefois des limitations importantes : souvent, elles sont de petite échelle et réalisées dans un seul centre, ce qui a pour résultat qu'elles manquent des contrôles et techniques en aveugle adéquats. Pour cette raison, leur potentiel de généralisation est limité. Feagan et associés, dans le premier examen rigoureux de telles stratégies, ont randomisé 30 hôpitaux dans lesquels soit une stratégie multimodale de conservation du sang était mise en place, soit les soins standards étaient continués (y 
compris l'utilisation de don autologue préalable dans environ $25 \%$ des cas) pour les patients de chirurgie orthopédique. ${ }^{16}$ Les auteurs ont découvert que l'intervention n'était que modestement réussie, en ce qu'elle a réduit l'exposition aux transfusions de sang allogène que d'environ $10 \%$ (de $26,1 \%$ à $16,5 \%$ ). Ainsi, cette étude a révélé que les stratégies multimodales de conservation du sang, bien qu'efficaces, ne le sont pas autant que les recommandations cliniques qui encouragent leur utilisation le présupposent.

\section{Les cliniciens}

Le principe de base de la médecine est, avant tout, de ne pas faire de mal. ${ }^{17}$ Par conséquent, la décision d'avoir recours à une modalité périopératoire de conservation du sang, quelle qu'elle soit, devrait, comme dans toute autre intervention médicale, se baser sur une analyse méticuleuse des risques et bénéfices. ${ }^{17}$ C'est pourquoi les praticiens de médecine périopératoire sont face à un dilemme. D'une part, les experts leur conseillent d'employer des stratégies multimodales de conservation du sang afin de réduire l'exposition des patients aux transfusions périopératoires de sang allogène. D'autre part, l'innocuité et l'efficacité des modalités de conservation du sang n'ont pas été testées de manière appropriée ; ainsi, nous ne savons pas si leurs risques, que ces modalités soient utilisées individuellement ou combinées, sont compensés par leurs bénéfices.

Etant donné qu'il n'existe pas de données fiables au sujet des profils risques/bénéfices des modalités de conservation du sang, comment s'explique le vaste consensus parmi les experts quant à leur utilisation continue? Nous pensons qu'un corollaire peut être tiré avec la manière dont les experts ont réagi à la menace de la maladie de Creutzfeldt-Jakob (vMCJ) sur l'approvisionnement en sang. Des études ont démontré que l'approche envers cette menace était fortement influencée par les événements catastrophiques de l'épidémie d'infection par le sang des années 1970 et 1980, ayant pour résultat l'adoption de mesures de sécurité présentant une rentabilité relativement élevée avant même la parution de données probantes définitives (c.-à-d. une approche de précaution). ${ }^{18,19}$ Quoique cette approche puisse être considérée comme appropriée afin de protéger la sécurité de l'approvisionnement en sang, nous pensons qu'elle est inappropriée pour la conservation périopératoire du sang, dans laquelle chaque modalité présente des risques inhérents qui peuvent être bien plus élevés que les risques actuels connus des transfusions de sang allogène.

\section{Le gant}

Afin de résoudre le dilemme des cliniciens, nous mettons au défi les chercheurs et les compagnies pharmaceutiques impliqués dans ce domaine de concevoir et mettre en ouvre des études cliniques randomisées contrôlées avec une puissance statistique adéquate afin de déterminer le profil risque/bénéfice des stratégies multimodales de conservation périopératoire du sang. Nous sommes conscients que cela sera difficile, étant donné que cela nécessitera de larges études complexes et coûteuses. Néanmoins, de telles études ont été réalisées dans d'autres domaines, ${ }^{20}$ et sont réalisées dans ce domaine également. ${ }^{21}$ Jusqu'à ce que de telles études soient mises en œuvre, il serait prudent que les cliniciens ayant recours aux modalités de conservation du sang informent exhaustivement leurs patients quant aux risques potentiels des modalités utilisées et stipulent clairement au patient que ces risques pourraient être plus élevés que les bénéfices potentiels liés au fait d'éviter les transfusions de sang allogène. En dépit de l'opinion des experts, il serait encore plus prudent de limiter l'utilisation de la plupart des modalités de conservation du sang au cadre de la recherche et ce, jusqu'à ce que leur profil risque/bénéfice soit clairement déterminé.

\section{References}

1 Blajchman MA, Vamvakas EC. The continuing risk of transfusion-transmitted infections. N Engl J Med 2006; 355: 1303-5.

2 Kleinman S, Caulfield T, Chan P, et al. Toward an understanding of transfusion-related acute lung injury: statement of a consensus panel. Transfusion 2004; 44 : 1774-89.

3 Spiess BD. Risks of transfusion: outcome focus. Transfusion 2004; 44(Suppl 12): 4S-14S.

4 Society of Thoracic Surgeons Blood Conservation Guideline Task Force; Ferraris VA, Ferraris SP, Saba $S P$, et al. Perioperative blood transfusion and blood conservation in cardiac surgery: the Society of Thoracic Surgeons and The Society of Cardiovascular Anesthesiologists clinical practice guideline. Ann Thorac Surg 2007; 83(5 Suppl): S27-S86.

5 Goodnough LT, Shander A. Blood management. Arch Pathol Lab Med 2007; 131: 695-701.

6 Mangano DT, Tudor IC, Dietzel C; Multicenter Study of Perioperative Ischemia Research Group; Ischemia Research and Education Foundation. The risk associated with aprotinin in cardiac surgery. N Engl J Med 2006; 354: 353-65.

7 Karkouti K, Beattie WS, Dattilo KM, et al. A propensity score case-control comparison of aprotinin and tranexamic acid in high-transfusion-risk cardiac surgery. 
Transfusion 2006; 46: 327-38.

8 Brown JR, Birkmeyer NJ, O'Connor GT. Meta-analysis comparing the effectiveness and adverse outcomes of antifibrinolytic agents in cardiac surgery. Circulation 2007; 115: 2801-13.

9 de Andrade JR, Frei D, Guilfoyle M. Integrated analysis of thrombotic/vascular event occurrence in epoetin alfa-treated patients undergoing major, elective orthopedic surgery. Orthopedics 1999; 22(1 Suppl): s113-8.

10 Burton $A$. Is it all over for erythropoietin? Lancet Oncol 2007; 8: 285.

11 Escobar GA, Cheng AM, Moore EE, et al. Stored packed red blood cell transfusion up-regulates inflammatory gene expression in circulating leukocytes. Ann Surg 2007; 246: 129-34.

12 Karkouti K, Wijeysundera DN, Yau TM, et al. The independent association of massive blood loss with mortality in cardiac surgery. Transfusion 2004; 44: 1453-62.

13 Karkouti K, Yau TM, Riazi S, et al. Determinants of complications with recombinant factor VIIa for refractory blood loss in cardiac surgery. Can J Anesth 2006; 53: 802-9.

14 Helm RE, Rosengart TK, Gomez M, et al.

Comprehensive multimodality blood conservation: 100 consecutive CABG operations without transfusion. Ann Thorac Surg 1998; 65: 125-36.

15 Moskowitz DM, Klein JJ, Shander A, et al. Predictors of transfusion requirements for cardiac surgical procedures at a blood conservation center. Ann Thorac Surg 2004; 77: 626-34.

16 Wong CJ, Vandervoort MK, Vandervoort SL, et al. A cluster-randomized controlled trial of a blood conservation algorithm in patients undergoing total hip joint arthroplasty. Transfusion 2007; 47: 832-41.

17 Singer M, Glynne P. Treating critical illness: the importance of first doing no harm. PLoS Med 2005; 2 : el67.

18 Wilson K, Graham I, Ricketts M, Dornan C, Laupacis $A$, Hebert $P$. Variant Creutzfeldt-Jakob disease and the Canadian blood system after the tainted blood tragedy. Soc Sci Med 2007; 64: 174-85.

19 Ponte ML. Insights into the management of emerging infections: regulating variant Creutzfeldt-Jakob disease transfusion risk in the UK and the US. PLoS Med 2006; 3: e342.

20 Rivers E, Nguyen B, Havstad S, et al. Early goal-directed therapy in the treatment of severe sepsis and septic shock. N Engl J Med 2001; 345: 1368-77.

21 Coats T, Hunt B, Roberts I, Shakur H. Antifibrinolytic agents in traumatic haemorrhage. PLoS Med 2005; 2: e64. 